\title{
NEW LOCALITY OF GENTIANA CRUCIATA L. IN THE STRZYŻOWSKIE FOOTHILLS (WESTERN CARPATHIANS)
}

\author{
Tomasz WóJCik, KRzYsztof PiąTeK
}

\begin{abstract}
T. Wójcik, Department of Ecology and Nature Protection, University of Rzeszów, Cicha 2 A, 35-326 Rzeszów, Poland, e-mail: antomi7@wp.pl K. Piątek, Jodłowa 15 A, 39-225 Jodłowa, Poland
\end{abstract}

(Received: September 16, 2014. Accepted: January 28, 2015)

\begin{abstract}
AвSTRACT. In 2006, ca. 60 individuals of Gentiana cruciata were found in the Strzyżowskie Foothills. Until recently, only one locality of the species in the area has been reported from Kołaczyce in the Wisłoka River valley by Knapp in the 19th century. The new locality is situated in the Wielopolka River valley on an S-SW-W slope with varied inclination. In total, 16 phytosociological relevés were taken with the use of the Braun-Blanquet method in patches comprising Gentiana cruciata. The patches were characterised by high proportions of species from the classes Molinio-Arrhenathereta, Festuco-Brometea, and Trifolio-Geranietea. Given the dominance of meadow species, the community was classified as a thermophilic fresh meadow from the alliance Arrhenatherion. An upward trend was noted in the analysed population, as the number of the individuals increased to ca. 160 in 2014.
\end{abstract}

KEY WORDS: Gentiana cruciata, endangered species, xerothermic grasslands, Wielopolka River valley, Strzyżowskie Foothills

\section{INTRODUCTION}

Gentiana cruciata L. has a Euro-Siberian occurrence range, covering southern and central Europe, Asia Minor, the Caucasus, Turkestan, and western Siberia (Meusel et al. 1978, Hultén \& Fries 1986, ZajĄC \& ZaJAC 2009). In Poland, the localities of this species are concentrated in the mountains and along the upland belt in the south of the country. The species also occurs in the lower Oder and Vistula regions, the Mazury Lake District, as well as in Pomerania and Wielkopolska. Its most frequent occurrence has been noted in the Eastern Carpathians, the Lublin Upland, and in the Sudeten Mountains. It has not been reported from central Poland (ZAJĄC \& ZAJĄC 2001). In the mountains, the species reaches the lower forest zone. The highest locality was reported from Wysokie Skałki in the Małe Pieniny Mountains (950 m a.s.1.) (PięKoś-Mirkowa \& Mirek 2003).

Gentiana cruciata belongs to the family Gentianaceae. It is a perennial species characterised by a thick rhizome and a height ranging from 10 to $40(60) \mathrm{cm}$.
It has an erect or rising, densely foliated stem with internodes that are considerably shorter than the leaves. The basal leaves, usually 5-8, are obovate-lanceolate in shape. The light-green stem leaves are fused into a sheath and exhibit decussate arrangement. They are lanceolate-obovate or lanceolate in shape and $10 \mathrm{~cm}$ long and $2 \mathrm{~cm}$ wide. The flowers are arranged in a capitulum at the stem apex; additionally, 1-3 sessile, tetramerous flowers cluster in the upper leaf axils. The $2-3 \mathrm{~cm}$ long corolla is tubular-campanulate in shape, tetragonal, dirty-blue in colour, with triangular, outwardly curved lobes. The fruit is a $2 \mathrm{~cm}$ elongated capsule with wingless seeds. The species flowers between June and August (JasieWiCZ 1971, PięKoś-MirkowA and Mirek 2003, 2006).

Gentiana cruciata is a hemicryptophyte with considerable light and thermal requirements (ZARZYCKI et al. 2002). It grows on dry meadows, grasslands, pastures, steep and warm slopes, in light scrubs, at forest edges, and along roadsides. It prefers clay or loess soils containing calcium carbonate with neutral or alkaline reaction ( $\mathrm{pH} 6,6-8,4)$. It occurs on 
carbonate rendzinas and pararendzinas, and less frequently on brown earth and chernozems proper (Pį̨Koś-Mirkowa \& Mirek 2003, 2006).

Gentiana cruciata is a characteristic species for the class Festuco-Brometea and, regionally, for the association Adonio-Brachypodietum pinnati (MATUSZKIEWICZ 2001). In the Małopolska Upland, it was noted in Sisymbrio-Stipetum capillatae patches (КоsтUсH \& MiszTAL 2007). It also grows in thermophilic meadow communities, e.g. on xerothermic Anthyllidi-Trifolietum montani meadows in the Pieniny Mountains (KaźmierCZAKOWA et al. 2004), in a thermophilic fresh meadow sub-association Arrhenatheretum elatioris brizetosum mediae in the Przemyskie Foothills (BARABASZ-KRASNY 2011), and in a mesophilic sub-association Adonio-Brachypodietum pinnati arrhenatheretosum and a community Centaurea scabiosa-Agrimonia eupatoria in the Silesian Upland (BABCZYŃSKA-SENDEK 2005).

In Poland, Gentiana cruciata is under strict species protection (RozPORZĄDZENIE... 2014). Moreover, it has been included in many regional red lists and assigned category CR in the Opole Voivodeship and Wielkopolska (BABCZYŃSKA-SENDEK \& NoWAK 2002, JACKOWIAK et al. 2007, NowAK et al. 2008); EN: in Gdańsk Pomerania and Western Pomerania (ŻukowSKI \& JACKOWIAK 1995, MARKOWSKI \& BULIŃSKI 2004); VU: in the Sudeten and the entire Lower Silesia, Silesian Province, Małopolska Upland, Roztocze, Western Polesie, Kuyavian-Pomeranian Province, Południowopodlaska Lowland, and Łodź Province (KucharczyK \& WójCiak 1995, RutKowsKi 1997, BerNACKI et al. 2000, FABISZEWSKI \& KWIATKOWSKI 2002, GŁowacki et al. 2003, KącKi et al. 2003, Bróż \& Przemyski 2009, Kurzac \& Olaczek 2012, Parusel \& URBISZ 2012). In the Świettokrzyskie Province and the northern part of the Silesian-Cracow Upland, the species has a status of a rare plant with category $\mathrm{R}$ (Bróż 1990, HereźniaK 2002).

\section{MATERIAL AND METHODS}

The investigations were conducted in two periods: 2006-2007 and 2012-2014. Sixteen phytosociological relevés were taken using the Braun-Blanquet method (PAwŁowski 1977), i.e. two in 2006-2007 and 14 in 2012-2013. The relevés are presented in a synthetic table (Table 1). Phytosociological stability and the coverage index were calculated. The species names follow a critical list of Polish vascular plants (MireK et al. 2002) and the syntaxonomic affiliation was determined according to the system proposed by Matuszkiewicz (2001).

\section{DESCRIPTION OF THE LOCALITY}

The newly discovered locality is situated in Nawsie at the border with Wielopole Skrzyńskie in the Podkarpackie Voivodeship. The area is located in the Strzyżowskie Foothills, which belongs to the Outer Western Carpathians (KoNDRACKI 2012). Until recently, Gentiana cruciata has been reported in the Strzyżowskie Foothills only in Kołaczyce in the Wisłoka River valley (KNAPP 1869, TowPASz 1987), and this locality still exists. In Nawsie, the species grows on the slope of an alluvial fan characterised by varied exposure and inclination. The fan is intersected by a $2 \mathrm{~m}$ deep holweg formed through erosion of a field path. It divides the locality into two parts. The part located below the holweg (S exposure) is currently a fallow that is becoming overgrown by Prunus spinosa. It is irregularly grazed or mowed only at its foot. The other part of the slope located above the holweg (SW-W exposure) is regularly grazed. An additional terrain form is an earth excavation pit formed in the lower part of the slope.

The analysed species was first noted in this area in 2006 and its occurrence was documented with a phytosociological relevé. The observation was repeated the following year. Detailed investigations were carried out in 2012-2014, when 14 phytosociological relevés, each with an area of $25 \mathrm{~m}^{2}$, were taken (Table 1). The relevés showed 23 to 41 species (31 on average). In the first period of the research, 60 Gentiana cruciata individuals were noted (50 below the holweg and 10 in the upper part of the slope). In the other period, 160 individuals were observed. A substantial increase in their number was noted on the grazed slope above the field path (95), while there were 65 individuals below the holweg. From two to seven shoots (on average four) were found in the scrubs. The community with Gentiana cruciata has a thermophilic nature and its species composition resembles that of fresh meadows, xerothermic grasslands, and thermophilic fringes. The patches comprised seven species from each of the Festuco-Brometea and Trifolio-Geranietea classes. Some of them are rare in the flora of the Strzyżowskie Foothills (Centaurea scabiosa) or they have been noted in only one locality in this part of the Carpathians (Brachypodium pinnatum, Scabiosa ochroleuca, Gentiana cruciata). All the relevés showed dominance of meadow species from the alliance Arrhenatherion (e.g. Arrhenatherum elatius, Galium mollugo), order Arrhenatheretalia (e.g. Achillea millefolium, Daucus carota), and class Molinio-Arrhenatheretea (e.g. Vicia cracca, Planatago lanceolata, Leontodon hispidus). Crataegus monogyna, Prunus spinosa, Rosa canina, Cornus sanguinea shrubs were noted in the uncultivated part of the slope. Their abundance evidences progressive succession towards scrubs from the class Rhamno-Prunetea. Additionally, species typical of ruderal habitats (e.g. Cichorium intybus, Cirsium vulgare, Melilotus officinalis), which encroached from the adjacent fallows and post-agricultural land, were found. The community with Gentiana cruciata was accompanied by species that do not belong to any syntaxonomic unit. 
Table 1. Occurrence of Gentiana cruciata in thermophilic meadow communities from the alliance Arrhenatherion

\begin{tabular}{|c|c|c|c|c|c|c|c|c|c|c|c|c|c|c|c|c|c|c|}
\hline $\begin{array}{l}\text { Succesive number of } \\
\text { relevé }\end{array}$ & 1 & 2 & 3 & 4 & 5 & 6 & 7 & 8 & 9 & 10 & 11 & 12 & 13 & 14 & 15 & 16 & \multirow{12}{*}{ 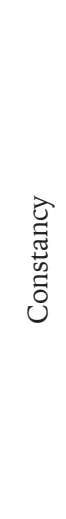 } & \multirow{12}{*}{ 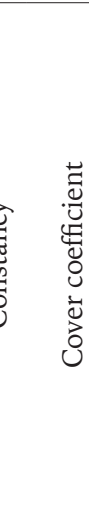 } \\
\hline day & 3 & 18 & 7 & 7 & 7 & 7 & 7 & 7 & 7 & 12 & 12 & 12 & 12 & 12 & 12 & 12 & & \\
\hline \multirow[t]{2}{*}{ Date } & 7 & 6 & 7 & 7 & 7 & 7 & 7 & 7 & 7 & 7 & 7 & 7 & 7 & 7 & 7 & 7 & & \\
\hline & year 2006 & 2007 & 2012 & 2012 & 2012 & 2012 & 2012 & 2012 & 2012 & 2013 & 2013 & 2013 & 2013 & 2013 & 2013 & 2013 & & \\
\hline Aspect & SW & SW & SW & W & SW & SW & SW & S & $S$ & $S$ & $S$ & $S$ & $S$ & $S$ & $S$ & S & & \\
\hline Inclination $\left[^{\circ}\right]$ & 30 & 30 & 20 & 30 & 30 & 30 & 35 & 45 & 10 & 40 & 40 & 40 & 40 & 5 & 10 & 35 & & \\
\hline Altiude [m a.s.l.] & 268 & 268 & 275 & 267 & 279 & 278 & 284 & 258 & 258 & 261 & 261 & 261 & 261 & 267 & 267 & \multirow{2}{*}{$\begin{array}{l}266 \\
100\end{array}$} & & \\
\hline Cover of herb layer $[\%]$ & 90 & 90 & 100 & 95 & 100 & 100 & 100 & 100 & 100 & 100 & 100 & 100 & 100 & 100 & 100 & & & \\
\hline Cover of shrub layer [\%] & - & - & - & - & - & - & - & - & 15 & - & - & - & - & 10 & 5 & - & & \\
\hline Management* & $\mathrm{g}$ & g & $\mathrm{g}$ & $\mathrm{g}$ & g & $\mathrm{g}$ & g & $\mathrm{g}$ & - & $\mathrm{m} / \mathrm{g}$ & $\mathrm{m} / \mathrm{g}$ & $\mathrm{m} / \mathrm{g}$ & $\mathrm{m} / \mathrm{g}$ & - & - & $\mathrm{m} / \mathrm{g}$ & & \\
\hline Area of relevé $\left[\mathrm{m}^{2}\right]$ & 100 & 100 & 25 & 25 & 25 & 25 & 25 & 25 & 25 & 25 & 25 & 25 & 25 & 25 & 25 & 25 & & \\
\hline Number of species & 26 & 32 & 41 & 35 & 23 & 28 & 26 & 29 & 27 & 40 & 38 & 32 & 33 & 25 & 30 & 32 & & \\
\hline Gentiana cruciata & 1.2 & 1.2 & 1.2 & + & + & 1.2 & 1.2 & r & 2.2 & + & + & r & r & 1.2 & + & + & $\mathrm{V}$ & 325 \\
\hline \multicolumn{19}{|l|}{ ChAll. Arrhenatherion } \\
\hline Arrhenatherum elatius & 2.2 & 2.2 & 3.2 & + & . & 2.2 & 3.2 & 5.5 & 3.3 & 3.2 & 2.2 & 2.2 & 3.2 & 2.2 & 2.2 & 1.2 & \multicolumn{2}{|c|}{ V 2519} \\
\hline Galium mollugo & . & 1.2 & 1.2 & + & + & + & + & + & 1.2 & 2.2 & 1.2 & + & + & 1.2 & 2.2 & . & $\mathrm{V}$ & 397 \\
\hline Knautia arvensis & . & + & + & + & . & + & 1.1 & 1.1 & + & + & + & . & + & + & + & + & $\mathrm{V}$ & 97 \\
\hline Crepis biennis & + & 1.2 & 1.2 & 1.2 & + & . & . & + & . & + & + & + & + & . & . & + & IV & 119 \\
\hline ChO. Arrhenatheretalia e & latior & & & & & & & & & & & & & & & & & \\
\hline Achillea millefolium & 2.2 & 1.1 & 1.1 & 2.2 & 4.3 & 1.2 & + & 1.1 & + & + & + & + & 1.1 & + & 1.2 & 1.2 & $\mathrm{~V}$ & 847 \\
\hline Daucus carota & + & . & + & 2.2 & 1.2 & + & + & 1.1 & . & + & + & + & 1.1 & + & + & 1.1 & V & 263 \\
\hline Dactylis glomerata & + & 1.2 & + & . & . & + & + & + & + & + & + & + & + & + & + & + & $\mathrm{V}$ & 72 \\
\hline Lotus corniculatus & . & + & + & + & . & + & + & . & + & + & + & + & . & . & . & + & IV & 31 \\
\hline Trifolium repens & . & + & + & + & + & . & . & . & . & . & . & . & . & . & . & . & II & 13 \\
\hline Bellis perennis & . & + & . & . & . & . & . & . & . & . & . & . & . & . & . & . & I & 3 \\
\hline Cynosurus cristatus & . & + & . & . & . & . & . & . & . & . & . & . & . & . & . & . & I & 3 \\
\hline Heracleum sphondylium & . & . & . & . & . & + & . & . & . & . & . & . & . & . & . & . & I & 3 \\
\hline
\end{tabular}

\section{ChCl. Molinio-Arrhenatheretea}

\begin{tabular}{|c|c|c|c|c|c|c|c|c|c|c|c|c|c|c|c|c|c|c|}
\hline \\
\hline Vicia cracca & . & . & + & + & + & + & + & + & + & + & + & + & + & + & + & + & V & 44 \\
\hline Plantago lanceolata & + & + & + & 1.2 & 1.2 & . & 1.1 & + & . & + & 1.2 & + & + & + & . & + & $\mathrm{V}$ & 153 \\
\hline Leontodon hispidus & + & 2.2 & + & 2.2 & 1.2 & 1.2 & 1.1 & + & . & + & + & . & + & . & . & . & IV & 331 \\
\hline Festuca rubra & . & . & + & . & . & . & . & +.2 & . & +.2 & 1.2 & 1.2 & 1.2 & 1.2 & 2.2 & . & III & 244 \\
\hline Trifolium pratense & + & + & + & + & . & + & +.2 & . & . & . & . & . & . & . & . & + & III & 22 \\
\hline Potentilla reptans & . & . & . & . & . & . & . & + & . & + & + & + & + & . & + & . & II & 19 \\
\hline Prunella vulgaris & . & + & + & + & . & . & 1.1 & . & . & . & . & . & . & . & . & + & II & 44 \\
\hline Festuca pratensis & . & . & . & . & . & . & . & . & . & . & +.2 & +.2 & +.2 & . & . & +.2 & II & 13 \\
\hline Phleum pratense & . & . & + & . & . & . & . & . & . & . & . & + & . & . & + & . & I & 9 \\
\hline Poa trivialis & . & . & + & . & + & . & . & + & . & . & . & . & . & . & . & . & I & 9 \\
\hline Lolium perenne & . & . & . & 2.2 & + & . & . & . & . & . & . & . & . & . & . & . & I & 113 \\
\hline Poa pratensis & . & . & . & . & + & . & . & . & . & . & + & . & . & . & . & . & I & 6 \\
\hline Centaurea jacea & . & . & + & . & . & . & . & . & . & + & . & . & . & . & . & . & I & 6 \\
\hline Rhinanthus angustifolius & . & . & +.2 & . & . & . & . & . & . & . & . & . & . & . & . & . & I & 3 \\
\hline Taraxacum officinale & . & + & . & . & . & . & . & . & . & . & . & . & . & . & . & . & I & 3 \\
\hline \multicolumn{19}{|l|}{ ChAll. Festuco-Brometea } \\
\hline Centaurea scabiosa & + & + & 2.3 & + & . & + & 2.2 & 3.3 & . & 1.2 & + & + & + & + & . & 1.1 & $\mathrm{~V}$ & 541 \\
\hline Poa compressa & . & . & + & +.2 & . & + & +.2 & . & + & + & 1.2 & 2.2 & + & + & + & + & IV & 172 \\
\hline Plantago media & 2.2 & 1.2 & + & + & 1.2 & 3.3 & . & . & 1.2 & . & . & . & . & + & + & 1.1 & IV & 481 \\
\hline Brachypodium pinnatum & . & . & . & . & . & . & . & +.2 & . & 1.3 & +.3 & +.3 & +.2 & . & . & . & II & 44 \\
\hline Allium oleraceum & . & . & . & + & . & . & . & . & . & . & . & + & . & + & . & + & II & 13 \\
\hline Scabiosa ochroleuca & 1.1 & . & . & . & . & . & . & . & . & . & . & . & . & . & . & . & I & 31 \\
\hline \multicolumn{19}{|l|}{ ChAll. Trifolio-Geranietea } \\
\hline Origanum vulgare & 3.2 & 1.2 & 3.3 & 3.2 & 1.2 & 1.2 & 2.2 & 1.2 & 3.3 & 2.2 & 3.2 & 4.3 & 3.2 & 4.3 & 4.3 & 3.2 & $\mathrm{~V}$ & 3156 \\
\hline
\end{tabular}




\begin{tabular}{lcccccccccccccccccc}
\hline Agrimonia eupatoria & 2.1 & 1.1 & 1.2 & 1.1 & + & 2.2 & 2.1 & + & 1.1 & 1.1 & 1.1 & 1.1 & + & 2.1 & + & 3.1 & V & 903 \\
Clinopodium vulgare &. &. & + & + & + & 1.2 &. & 2.2 & 1.2 & 1.2 & 1.2 & + & 1.2 & 2.2 & 2.2 &. & IV & 497 \\
Coronilla varia & 1.2 & 1.2 & + & + &. & + &. & + & + & + & + & + & + &. &. & + & IV & 94 \\
Medicago falcata & 1.1 & + & + & + & + & 3.3 & 1.1 &. & + &. &. &. &. &. & + &. & III & 316 \\
Galium verum &. &. & + &. &. & + &. &. & + &. &. &. &. &. &. &. & I & 9 \\
Astragalus glycyphyllos &. &. &. &. &. &. &. &. &. &. &. &. &. &. &. & + & I & 3
\end{tabular}

$\mathrm{ChCl}$. Artemisietea vulgaris

Cichorium intybus

Cirsium vulgare

Melilotus officinalis

Carduus acanthoides

Cirsium arvense

Chaerophyllum aroma-

ticum

Linaria vulgaris

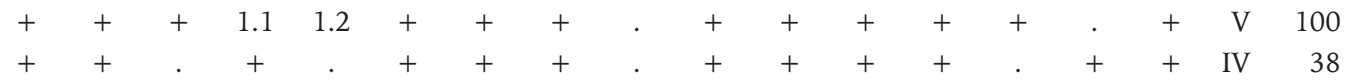

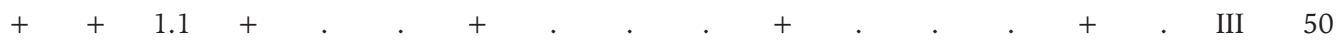

$$
\begin{aligned}
& \text { III } 13 \\
& \text { III } 13 \\
& \text { I } 3
\end{aligned}
$$

\section{ChCl. Rhamno-Prunetea}

Crataegus monogyna

Crataegus monogyna (b)

Prunus spinosa

Prunus spinosa (b)

Rosa canina

Rosa canina (b)

Cornus sanguinea

\begin{tabular}{|c|c|c|c|c|c|c|c|c|c|c|c|c|c|c|c|c|c|c|}
\hline Ononis arvensis & + & + & + & + & 4.4 & + & & . & & + & & . & 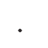 & . & . & + & III & 413 \\
\hline Briza media & + & + & + & . & . & . & . & . & 2.2 & . & . & +.2 & . & . & + & 2.2 & III & 234 \\
\hline Sanguisorbia minor & + & 1.2 & . & . & . & . & . & . & + & + & 1.1 & + & + & + & + & . & III & 84 \\
\hline Medicago lupulina & + & 1.2 & . & . & . & . & . & . & . & + & + & + & + & + & . & + & III & 53 \\
\hline Convolvulus arvensis & + & . & + & . & + & . & . & + & . & + & + & + & + & . & . & + & III & 28 \\
\hline Ranunculus polyanthemos & . & + & + & + & +.2 & + & + & + & . & . & . & . & . & . & . & . & III & 22 \\
\hline Euphorbia esula & . & . & + & + & . & . & . & + & . & . & + & + & + & . & . & + & III & 22 \\
\hline Mentha arvensis & . & . & . & . & +.2 & 1.2 & . & 2.2 & 1.2 & . & . & . & . & . & . & . & II & 175 \\
\hline Hypericum perforatum & 1.2 & + & . & . & . & . & . & + & + & . & . & . & . & + & + & . & II & 47 \\
\hline Fragaria vesca & . & . & . & . & . & . & . & . & + & . & . & . & . & 1.2 & + & . & I & 38 \\
\hline Pimpinella saxifraga & . & . & + & + & . & . & . & . & . & + & . & . & . & . & + & + & II & 16 \\
\hline Erigeron acre & . & . & . & . & . & . & . & +.2 & . & + & + & . & + & . & . & . & II & 13 \\
\hline Linum catharticum & . & 1.1 & . & + & . & . & . & . & . & . & . & . & . & . & . & + & I & 38 \\
\hline Equisetum arvense & . & . & . & + & . & . & 1.1 & . & . & . & . & . & . & . & . & + & I & 38 \\
\hline Erigeron annuus & . & . & . & . & 1.1 & . & . & . & . & . & + & . & . & . & . & . & I & 34 \\
\hline Calamagrostis epigejos & . & . & . & . & . & . & . & . & 1.2 & . & . & . & . & . & . & . & I & 31 \\
\hline Carex spicata & . & . & . & . & . & . & . & . & . & . & + & . & + & . & + & . & I & - \\
\hline Elymus repens & . & . & . & . & . & . & . & . & . & + & . & . & + & . & + & . & I & 9 \\
\hline Senecio jacobaea & . & . & + & + & . & . & . & . & . & . & . & . & . & . & . & + & I & 9 \\
\hline Holcus mollis & . & . & + & . & . & . & . & . & . & . & . & . & . & . & + & . & I & 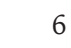 \\
\hline Medicago xvaria & . & . & . & . & . & . & . & . & . & + & + & . & . & . & . & . & I & 6 \\
\hline Quercus robur (b) & . & . & . & . & . & . & . & . & . & . & . & . & . & + & + & . & I & 6 \\
\hline Rubus caesius & . & . & + & $\cdot$ & . & . & . & . & . & + & . & . & $\cdot$ & . & . & . & I & 6 \\
\hline Thymus pulegioides & . & . & +.2 & . & . & . & . & . & . & . & . & . & . & . & . & . & I & 3 \\
\hline Pyrus pyraster (b) & . & . & . & . & . & . & . & . & + & $\cdot$ & . & . & . & . & . & . & I & 3 \\
\hline Pyrus pyraster & . & . & . & . & . & . & + & . & . & . & . & . & . & . & . & . & I & 3 \\
\hline Bromus inermis & . & . & . & . & . & . & . & . & . & + & . & . & $\cdot$ & . & . & . & I & 3 \\
\hline Mediacago sativa & . & . & . & . & . & . & . & . & . & . & + & . & . & . & . & . & I & 3 \\
\hline Cuscuta epithymum & . & . & . & . & . & . & . & . & . & + & . & . & . & . & . & . & I & 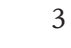 \\
\hline Stellaria graminea & + & . & . & . & . & . & . & . & . & . & . & . & . & . & . & . & I & \\
\hline
\end{tabular}

Cornus sanguinea (b)

\section{Others}

*Management: g - grazing, m/g - mowing and grazing. 
These included many plants inhabiting dry and insolated sites, e.g. Briza media, Sanguisorba minor, Ranunculus polyanthemos, and Pimpinella saxifraga.

\section{DISCUSSION AND CONCLUSIONS}

Gentiana cruciata is one of the characteristic species of the class Festuco-Brometea, although it can frequently be found in moderately or weakly xerothermic plant communities. In the Pieniny Mountains, the species is widespread across the entire region, where it occurs in various types of communities: from dry pastures and thermophilic meadows, xerothermic grasslands, and roadsides to thermophilic beech forests (KAŹMIERCZAKOWA 2004, KaźMIERCZAKOWA et al. 2004, Frey \& Tybur 2012). In the Silesian Upland, the species inhabits more mesophilic grasslands from the sub-association Adonio-Brachypodietum pinnati arrhenatheretosum and the community Centaurea scabiosa-Agrimonia eupatoria (BABCZYŃSKA-SENDEK 2005). It has also been noted in communities that comprise, besides species from the class Festuco-Brometea, great numbers of meadow species from the class Molinio-Arrhenatheretea and fringe species from the class Trifolio-Geranietea. These communities include poorly developed xerothermic grasslands formed in post-agricultural habitats, disused quarries, embankments, and roadsides (BABCZYŃSKA-SENDEK \& ANDRZEJCZUK 1997, BABCZYŃSKA-SENDEK et al. 2014).

In the area of the Strzyżowskie Foothills, there are no typical xerothermic grasslands as those occurring in other regions of Poland. Therefore, the presence of thermophilic plant patches deserves special attention. In the community with Gentiana cruciata, seven species from each of the classes Festuco-Brometea and Trifolio-Geranietea were found. Sites with such abundance of xerothermic plants are difficult to find in the analysed area. It is clearly dominated by meadow species from the class Molinio-Arrhenatheretea, since as many as 27 of these were found. The analysed phytocoenoses should be therefore classified as a thermophilic fresh meadow from the alliance Arrhenatherion. Occurrence of Gentiana cruciata in meadow communities Arrhenatheretum elatioris brizetosum mediae was also observed in the Przemyskie Foothills. The phytocoenoses with its share were characterised by high species richness and abundance of species from the classes Trifolio-Geranietea and Festuco-Brometea (BARABASZ-KRASNY 2011). Gentiana cruciata can also be found in the thermophilic Pieniny meadow Anthyllidi-Trifolietum montani associated with extensive land use (KAźmiercZAKOWA et al. 2004).

In many regions of Poland, Gentiana cruciata grows on poorly developed xerothermic grasslands, which may be related to destruction or abandonment thereof. More frequently, the species can be found in secondary post-agricultural and post-mining habitats as well as along roadsides (BABCZYŃSKA-SENDEK \& AN-
DRZEJCZUK 1997, BABCZyŃSKa-SENDEK et al. 2014, Heise 2010). The emergence of new localities of the species in secondary habitats indicates its long distance dispersal capability (BABCZYŃSKA-SENDEK et al. 2014).

In the area of the Foothills, xerothermic vegetation patches were regarded as part of thermophilic fresh meadows from the alliance Arrhenatherion (TOWPASZ 1990, BARABASZ-KRASNY 2011). Recently, a report on a Natura 2000 site in the Dynowskie Foothills titled "Łąki nad Wojkówką" has been published. The site comprises small patches of thermophilic vegetation with species composition resembling that of fresh meadows, xerothermic grasslands, and fringes. The communities described therein included a community from the class Trifolio-Geranietea, a community with Hieracium bauhinii, a community with Melampyrum arvense-Salvia verticillata, and a community with Centaurea scabiosa. Gentiana cruciata is extremely rare in this area and inhabits phytocoenoses from the class Trifolio-Geranietea (ZIAJA \& WójcıK 2014).

In many regions of Poland, Gentiana cruciata is losing its localities and is regarded as a retreating species (BABCZyŃSKa-SENDEK \& ANDRZEJCZuK 1997, BABCZYŃSKA-SENDEK \& NOWAK 2002, BABCZYŃSKA-SENDEK 2005). The greatest threat is posed by abandonment of traditional forms of land use, which triggers successional changes in vegetation. Equally unfavourable are changes in grassland management forms, such as ploughing and afforestation, which lead to habitat loss (Pięroś-Mirkowa \& Mirek 2003). Gentiana cruciata was observed to disappear in sites occupied by competitive expansive (Calamagrostis epigejos) or invasive (Solidago gigantea) perennial species and some shrub species (Prunus spinosa) (BABCZYŃSKa-SENDEK \& AndRZEjCZuK 1997, BABCZYŃSKA-SENDEK et al. 2014). Another threat is the small population size and the inconsiderable surface area of grassland phytocoenoses comprising the species. The new Gentiana cruciata locality covers an area of approx. $0.5 \mathrm{ha}$, but the abundance of the species exhibits an upward trend, since the population size has increased from ca. 60 to ca. 160 individuals in several years. Undoubtedly, the grazing management in the upper part of the slope is a beneficial factor, as it limits the growth of expansive plant species.

The xerothermic grasslands in the Strzyżowskie Foothills are rare, fragmentary communities with occurrence limited to river valleys. Detailed research should be undertaken to provide profound knowledge and full characterisation thereof.

\section{REFERENCES}

BabCZyŃsKa-Sendek B. (2005): Problemy fitogeograficzne i syntaksonomiczne kserotermów Wyżyny Śląskiej. Wyd. Uniwersytetu Śląskiego, Katowice.

BabczyŃsKA-Sendek B., AndrzejCZuk I. (1997): Goryczka krzyżowa Gentiana cruciata w okolicach 
Tarnowskich Gór. Natura Silesiae Superioris 1: 33-42.

BABczyńsKa-Sendek B., Błońska A., Skowronek I. (2014): Nowe stanowiska Gentiana cruciata (Gentianaceae) na Wyżynie Krakowsko-Częstochowskiej. Fragmenta Floristica et Geobotanica Polonica 21(1): 67-76.

BABCZyŃSKa-SendeK B., NowaK A. (2002): Goryczka krzyżowa. In: A. Nowak, K. Spałek (eds). Czerwona księga roślin województwa opolskiego. Rośliny naczyniowe wymarłe, zagrożone i rzadkie. Opolskie Towarzystwo Przyjaciół Nauk, Śląskie Wydawnictwo ADAN, Opole: 34.

Barabasz-Krasny B. (2011): Zróżnicowanie roślinności i sukcesja wtórna na odłogach wielkopowierzchniowych Pogórza Przemyskiego. W. Szafer Institute of Botany, Polish Academy of Sciences, Kraków.

Bernacki L., Nowak T., Urbisz A., Urbisz A., Tokarska-Guzik B. (2000): Rośliny chronione, zagrożone i rzadkie we florze województwa śląskiego. Acta Biologica Silesiana 35(52): 78-107.

Bróż E. (1990): Lista wymierających i zagrożonych gatunków roślin naczyniowych Krainy Świętokrzyskiej. Rocznik Świętokrzyski 17: 97-105.

Bróż E., Przemyski A. (2009): The red list of vascular plants in the Wyżyna Małopolska Upland (S Poland). In: Z. Mirek, A. Nikiel (eds). Rare, relict and endangered plants and fungi in Poland. W. Szafer Institute of Botany, Polish Academy of Sciences, Kraków: 123-136.

FABIsZEwski J., KWIATKOwSKi P. (2002): Threatened vascular plants of the Sudeten Mountains. Acta Societatis Botanicorum Poloniae 71(4): 339-350.

Frey L., Tybur J. (2012): Atlas roślin pienińskich. Kwiaty św. Kingi. Instytut Botaniki im. W. Szafera, Polska Akademia Nauk, Kraków.

GŁowacki Z., Falkowski M., Krechowski J., Marciniuk J., Marciniuk P., Nowicka-Falkowska K., Wierzba M. (2003): Czerwona lista roślin naczyniowych Niziny Południowopodlaskiej. Chrońmy Przyrodę Ojczystą 59(2): 5-41.

HeISE W. (2010): Murawy kserotermiczne doliny Kanału Bydgoskiego - rozmieszczenie wybranych gatunków rzadkich, zarys historii użytkowania i perspektywy zachowania. In: H. Ratyńska, B. Waldon (eds). Ciepłolubne murawy w Polsce stan zachowania i perspektywy ochrony. Wyd. Uniwersytetu Kazimierza Wielkiego, Bydgoszcz: 171-183.

HeREŹNIAK J. (2002): Regionalna lista wymarłych i zagrożonych gatunków roślin naczyniowych północnej części Wyżyny Śląsko-Krakowskiej. Acta Universitatis Lodziensis, Folia Biologica et Oecologica 1: 39-63.

Hultén E., Fries M. (1986): Atlas of North European vascular plants: north of the Tropic of Cancer, II. Koeltz Scientific Books, Königstein.
Jackowiak B., Celka Z., Chmiel J., Latowski K., ŻuKOWsKI W. (2007): Red list of vascular flora of Wielkopolska (Poland). Biodiversity Research and Conservation 5-8: 95-127.

JASIEWICZ A. (1971): Rodzina: Gentianaceae, Goryczkowate. In: B. Pawłowski, A. Jasiewicz (eds). Flora Polska. Vol. 12. Rośliny naczyniowe Polski i ziem ościennych. PWN, Warszawa-Kraków: 7-32.

KAźMIerCZAKOWA R. (2004): Kserotermiczne murawy i zarośla Pienińskiego Parku Narodowego. In: R. Kaźmierczakowa (ed.). Charakterystyka i mapa zbiorowisk roślinnych Pienińskiego Parku Narodowego. Polska Akademia Nauk, Instytut Ochrony Przyrody. Studia Naturae 49: 277-296.

Kaźmierczakowa R., Zarzycki J., Wróbel I., VonČIna G. (2004): Łąki, pastwiska i zbiorowiska siedlisk wilgotnych Pienińskiego Parku Narodowego. In: R. Kaźmierczakowa (ed.). Charakterystyka i mapa zbiorowisk roślinnych Pienińskiego Parku Narodowego. Polska Akademia Nauk, Instytut Ochrony Przyrody. Studia Naturae 49: 195-251.

KĄCKI Z., DAJDOK Z., SZCZĘ́́NIAK E. (2003): Czerwona lista roślin naczyniowych Dolnego Śląska. In: Z. Kącki (ed.). Zagrożone gatunki flory naczyniowej Dolnego Śląska. Instytut Biologii Roślin Uniwersytetu Wrocławskiego, Polskie Towarzystwo Przyjaciół Przyrody „pro Natura”, Wrocław: 9-65.

KnAPP A. (1869): Przyczynek do flory obwodów jasielskiego i sanockiego. Sprawozdania Komisji Fizjograficznej 3: 74-109.

KondRACKI J. (2012): Geografia regionalna Polski. Wyd. Nauk. PWN, Warszawa.

Kostuch R., Misztal A. (2007): Roślinność kserotermiczna istotnym elementem bioróżnorodności Wyżyny Małopolskiej. Woda-Środowisko-Obszary Wiejskie 7, 2b (21): 99-110.

KuCHARCZyK M., WójCiAK J. (1995): Ginące i zagrożone gatunki roślin naczyniowych Wyżyny Lubelskiej, Roztocza, Wołynia Zachodniego i Polesia Lubelskiego. Ochrona Przyrody 52: 33-46.

Kurzac M., Olaczer R. (2012): Gentiana cruciata L. goryczka krzyżowa. In: R. Olaczek (ed.). Czerwona księga roślin województwa łódzkiego. Ogród Botaniczny w Łodzi, Uniwersytet Łódzki, Łódź: 96-97.

Markowski R., Buliński M. (2004): Ginące i zagrożone rośliny naczyniowe Pomorza Gdańskiego. Acta Botanica Cassubica Monographiae 1: 1-75.

Matuszkiewicz W. (2001): Przewodnik do oznaczania zbiorowisk roślinnych Polski. Wyd. Nauk. PWN, Warszawa.

Meusel H., Jäger E., Rauschert S., Weinert E. (1978): Vergleichende Chorologie der zentraleuropäischen Flora. Bd. II. Gustav Fischer Verlag, Jena.

Mirek Z., Pięroś-Mirkowa H., Zając A., Zając M. (2002): Flowering plants and pteridophytes of Poland. A checklist. Vol. 1. Biodiversity of Poland. 
W. Szafer Institute of Botany, Polish Academy of Sciences, Kraków.

Nowak A., Nowak S., Spazek K. (2008): Red list of vascular plants of Opole province. Opole Scientific Society Nature Journal 41: 141-158.

Parusel J.B., Urbisz A. (eds) (2012): Czerwona lista roślin naczyniowych województwa śląskiego. In: J.B. Parusel (ed.). Raporty Opinie 6. Strategia ochrony przyrody województwa śląskiego do roku 2030. Raport o stanie przyrody województwa śląskiego 2. Czerwone listy wybranych grup grzybów i roślin województwa śląskiego. Centrum Dziedzictwa Przyrody Górnego Śląska, Katowice: 103-177.

PAwŁowsKi B. (1977): Skład i budowa zbiorowisk roślinnych oraz metody ich badania. In: W. Szafer, K. Zarzycki (eds). Szata roślinna Polski. Tom 1. Instytut Botaniki im. W. Szafera, Polska Akademia Nauk, Kraków: 237-269.

Piękoś-Mirkowa H., Mirek Z. (2003): Flora Polski. Atlas roślin chronionych. Multico Oficyna Wydawnicza, Warszawa.

PięKoś-Mirkowa H., Mirek Z. (2006): Flora Polski. Rośliny chronione. Multico Oficyna Wydawnicza, Warszawa.

RozPORZĄDZENIE Ministra Środowiska z dnia 9 października 2014 r. w sprawie ochrony gatunkowej roślin. Dz.U. z dnia 16 października 2014 r., poz. 1409.

Rutkowski L. (1997): Rośliny naczyniowe - Tracheophyta. In: J. Buszko, K. Kasprzyk, T. Pawlikowski, A. Przystalski, L. Rutkowski (eds). Czerwona lista roślin i zwierząt ginących i zagrożonych w regionie kujawsko-pomorskim. Acta Universitatis Nicolai Copernici, Biologia 53 (Suppl.), Nauki Matematyczno-Przyrodnicze 98: 5-20.
Towpasz K. (1987): Rośliny naczyniowe Pogórza Strzyżowskiego. Zeszyty Naukowe Uniwersytetu Jagiellońskiego, Prace Botaniczne 16: 1-160.

Towpasz K. (1990): Charakterystyka geobotaniczna Pogórza Strzyżowskiego. Zeszyty Naukowe Uniwersytetu Jagiellońskiego, Rozprawy habilitacyjne 178: 1-242.

ZaJĄC A., ZaJĄC M. (eds) (2001): Distribution atlas of vascular plants in Poland. Laboratory of Computer Chorology, Institute of Botany, Jagiellonian University, Kraków.

ZAJĄC M., ZAJĄC A. (2009): The geographical element of native flora of Poland. Laboratory of Computer Chorology, Institute of Botany, Jagiellonian University, Kraków.

Zarzycki K., TrzcińsKa-TaciK H., Różański W., SzeLĄG Z., WoŁeK J., Korzeniak U. (2002): Ecological indicator values of vascular plants of Poland. W. Szafer Institute of Botany, Polish Academy of Sciences, Kraków.

ZiAja M., WóJcıK T. (2014): Thermophilic plant communities in Natura 2000 site "Łąki nad Wojkówką” PLH 180051 - Podkarpacie Province. Annales Universitatis Mariae Curie-Skłodowska, Sectio C, Biologia 69(1): 59-78.

ŻuKowsKi W., Jackowiak B. (1995): Lista roślin naczyniowych ginących i zagrożonych na Pomorzu Zachodnim i w Wielkopolsce. In: W. Żukowski, B. Jackowiak (eds). Ginące i zagrożone rośliny naczyniowe Pomorza Zachodniego i Wielkopolski. Prace Zakładu Taksonomii Roślin UAM w Poznaniu 3. Bogucki Wyd. Nauk., Poznań: 9-96.

For citation: WóJCiK T., PiąTeK K. (2015): New locality of Gentiana cruciata L. in the Strzyżowskie Foothills (Western Carpathians). Steciana 19(2): 67-73. DOI 10.12657/steciana.019.008 